\title{
Frameless
}

Volume 1 | Issue 1

Article 28

November 2019

\section{Symposium Program}

Joe Geigel

Rochester Institute of Technology, jmg@cs.rit.edu

David Long

Rochester Institute of Technology, david.long@rit.edu

Follow this and additional works at: https://scholarworks.rit.edu/frameless

\section{Recommended Citation}

Geigel, Joe and Long, David (2019) "2016 Symposium Program," Frameless: Vol. 1: Iss. 1, Article 28.

DOI: 10.14448/Frameless.01.015

Available at: https://scholarworks.rit.edu/frameless/vol1/iss1/28

This Archives is brought to you for free and open access by RIT Scholar Works. It has been accepted for inclusion in Frameless by an authorized editor of RIT Scholar Works. For more information, please contact ritscholarworks@rit.edu. 


$$
\text { FRAMELESS }
$$

$$
\text { LABS }
$$

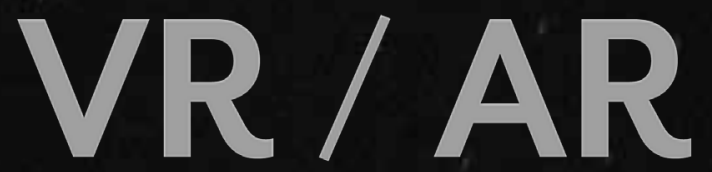

Symposium

$$
\text { DEC 2, } 2016
$$




\section{WELCOME!}

Frameless Labs is a newly formed organization on campus with a goal to bring together all research, innovation, and artistic creations surrounding the mediums of Virtual Reality and Augmented Reality. These efforts exist within many of the diverse colleges and departments housed under the brick roof of RIT. VR and AR are frameless mediums and open the window to a limitless sort of applications for science, education, content creation, and many more. The inaugural Frameless Labs VR/AR Symposium is the kick off event to link these efforts and share them with the RIT community.

Today's events are brought to you by Frameless Labs and MAGIC at RIT.

Introduction

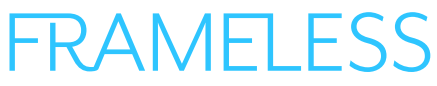

LABS

\section{SCHEDULE}

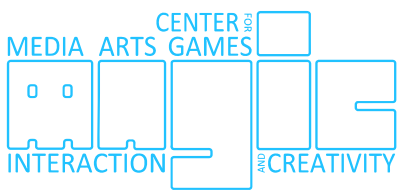

$10: 00-10: 15$

$10: 15-10: 30$

$10: 30-11: 00$
Coffee and arrival

Welcome (Dr. Jeremy Haefner)

Travels in the Uncanny Valley:

A Keynote by Flip Phillips

Talks/Presentations

11:00 - 12:00

$12: 00-1: 00$

$1: 00-1: 45$

$1: 45-2: 45$

$2: 45-3: 00$

$3: 00-4: 30$

$4: 30-5: 30$

$5: 30-6: 30$
SCIENCE (3 presentations)

Break

AR/VR Interdisciplinary Panel

CONTENT CREATION (4 presentations)

Break

TECH / INDUSTRY (5 presentations)

Social Hour: Refreshments and Demos

STORYTELLING \& PERFORMANCE 


\section{DEMONSTRATIONS \& POSTERS}

\section{Lunchtime Demos}

12:00 - 1:00

PerForM Lab in Imaging Science

(CAR-2101)

Modeling human hand-eye coordination using VR

Eye tracking during Obstacle avoidance using AR

Munsell Color Science Lab (MCSL)

(COL-1086)

Modeling Color Appearance in AR

CIAS New Media Design Workshop $\quad$ (BOO - 1320)

Experience Mindfulness

\section{Afternoon Demos}

1:45-5:00

VIVE Demos

Oculus Demos

Hololens \& VIVE

Posters

(MAGIC VR Lab in MAGIC Center)

(Coaching Room A in MAGIC Center)

(GOL 2400)

(GOL 2500)

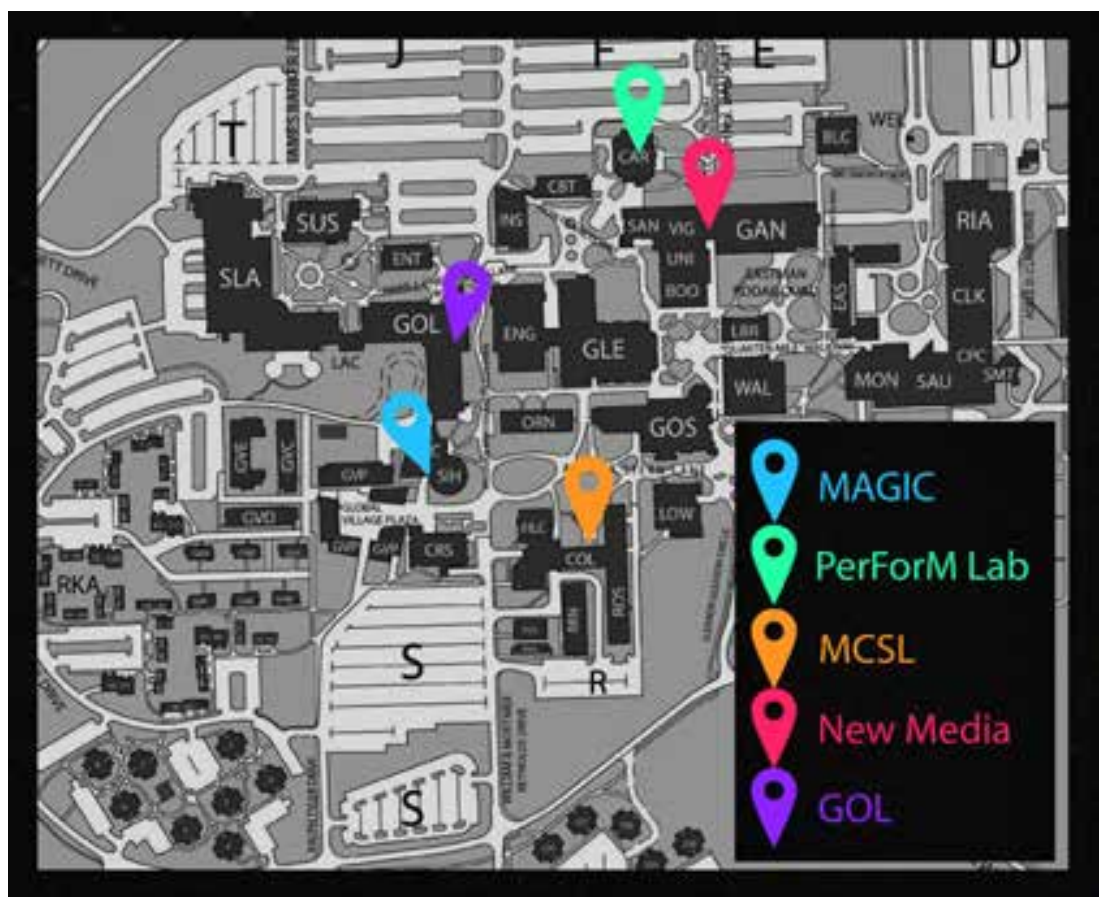




\section{KEYNOTE}

$10: 30-11: 00$

\section{Travels in the Uncanny Valley}

Flip Phillips, Skidmore College

In 1970, Masahiro Mori posited the existence of an "uncanny valley" in the human perception and appreciation of robotics. This hypothesis suggests that, as robots become more 'human like' that our appreciation of them increases - right up to a crucial point where we find them *too* realistic but not *perfectly* so. At this point, human preference plummets into the aforementioned chasm. Despite the fact that much has been written about this phenomena, there is little scientific evidence of its existence. Obviously this phenomena isn't limited to robotics and has been observed in many other areas. These include the fine arts, especially in photorealistic painting, sculpture, computer graphics, traditional animation, and more recently in the rotoscoping / motion capture used in contemporary filmmaking. The informal and heuristic practices of the fine arts, ${ }^{*}$ especially* those of traditional animation have much to offer to our understanding of the appearance of reality. In this talk, we will examine some historical perspective of the UV as well as virtual and augmented reality-centric questions.

\section{SCIENCE}

$11: 00-12: 00$

\section{Modeling Color Appearance in Augmented Reality}

\section{Nargess Hassani}

Color appearance in augmented reality is impacted by the background, lighting, and virtual appearance. By changing any of these factors, resultant color appearance can be different. In this project, we model and measure the color appearance in augmented reality.

\section{What you hear is what you see:}

\section{A new spatial ear-training paradigm on an AR device}

\section{Justin Levine}

A new spatial ear-training paradigm is proposed using a see-through head-mount display that overlays target visual objects over auditory objects. The training program engages trainees with a game that asks them to search for hidden visual objects based on auditory cues. This new paradigm present two significant merits that (1) a target object position is not limited by number of loudspeakers and (2) listeners can directly indicate the perceived location, which reduces the cognitive loads. 


\section{Modeling Human Hand-eye Coordination Using Virtual Reality}

\section{Kamran Binaee}

In this talk we present a research project that uses Virtual Reality to study human hand-eye coordination. Previous studies show that humans efficiently formulate predictive strategies to make accurate eye/ hand movements when intercepting with a target flying in their field of view. Nevertheless, it is not clear how these strategies tolerate noisy visual information such as occlusion. We used a VR ball catching paradigm to record the 3D gaze of ten subjects as well as their head and hand movements. We used machine learning techniques to model gaze and hand movements. This approach provides a flexible tool to model hand-eye coordination and investigate different hypotheses by comparing a model's performance with the human as ground truth.

\section{AR/VR INTERDISCIPLINARY PANEL AND EXHIBITION}

\section{$1: 00-1: 45$}

\section{Susan Lakin, David Halbstein, Joe Geigel, Josh Owen, Nitin Sampat}

An interdisciplinary panel of faculty collaborators will discuss emerging technology and curriculum development. The panel will address the challenges of integrating new technologies into the classroom and present documentation of successful student projects. In addition to the panel discussion, an exhibition of photographic prints with AR student content will be on display for audience engagement.

\section{CONTENT CREATION}

$1: 45-2: 45$

The Good the Bad and the Ugly, A VR 360 Perspective

Shaun Foster \& Alejandro Perez

Virtual Reality has the potential to become a widely adopted technology in the near future. This talk analyzes several aspects of "state of the art" VR. We will investigate the spectrum of what is amazing, what problems still exist and various "ugly" cultural elements which the new technology may open the doors to.

\section{Story Presence: A Scientific and Technical Approach to Narrative VR}

\section{Anna Dining}

Everyone is talking about VR and its potential as a storytelling medium. Many creatives are excited and want to dive right in. Yet we need more people thinking about optimal usage of VR and 360 video within the entertainment industy. This talk will explore how to categorize immersive storytelling experiences and then test controlled immersive and story variables for their effectiveness in inducing story presence. 


\section{Personalized Learning Environment using AR to Increase Student Engagement and Success}

\section{David Halbstein}

A personalized learning system that uses $A R$ and mobile devices to bring textbooks to life with additional web-based content. As students engage with their textbooks via AR; images, animations, language translations, and gamified formative assessments are provided to increase understanding of the material. Back-end analytics track student progress and report to an instructor dashboard, acting as an intermediary mentor to help faculty respond to both advanced and remedial students.

\section{We're All Living in the Gutter Now: "Storying the World" with AR and MR}

\section{Laura Shackelford}

A talk about fictional world-building as it encounters new AR/VR technologies with a few examples to illustrate how writers explore the storytelling potential of these technologies and critically examine what these technologies contribute to perception, interacting, and living in increasingly mixed-reality worlds.

\section{TECH \& THE INDUSTRY}

$3: 00-4: 30$

\section{Fly Safe Fix wing FPV}

\section{Bob Yang}

First Person Video Aviation has became a new fast growing part of RC model hobby. With its growth also comes safety and difficulty concerns. The goal of this project is to develop a type of small, medium performance, beginner friendly and crash resistant FPV fix wing platform for both flying line of sight and short range FPV flying.

\section{Creating Google VR In Unity}

\section{Anthony Zalar}

How to set up Google VR for video games. The presentation will prepare users for Android development with a focus on the Unity game engine.

\section{Interface.Server}

\section{Charlie Roberts}

This talk will describe ongoing research creating the interactive architecture for the AlloSphere, a three-story VR environment at UC Santa Barbara featuring twenty-six projectors covering the inside of a sphere and sixty-five speakers providing immersive audio. Up to forty users can stand on the bridge of the AlloSphere providing unique opportunities and challenges for interaction design. 


\section{Facebook Surround 360}

\section{Nick Franco}

Nick Franco, Kirsten Martin, and Madison Zic from CIAS Photographic Sciences have been working with Professor Nitin Sampat to research and develop ways of creating and editing 3D 360 content. In particular, they have been focusing on the Facebook Surround 360 camera. Most of their work pertains to what Facebook has already published as open source, and how to utilize and improve on those plans.

\section{Entrepreneurship in Virtual Reality}

\section{Justin Callaghan}

Innovators Hour's members are developing three projects in the VR and AR space. We will be speaking about these three projects specifically as well as their broader implication in VR and AR. These three projects are a VR headset designed for fitness, a wearable VR interface, and a non-obtrusive streamlined real time speak to text device.

\section{STORYTELLING \& PERFORMANCE}

\section{$5: 30-6: 30$}

\section{Farewell to Dawn: A Mixed Reality Dance Performance}

\section{Chirag Chandrakant Salian, Anna Dining, Joe Geigel, Rasmi Mukula} Kapuganti, Victoria McGowen, Zhongyuan Fa Anastasia Pembrook, Peter Ferry, Marla Schweppe, Steven Cerqueira, Quincey Williams

FAREWELL TO DAWN is a mixed-reality dance performance achieved using the Mixed Reality Stage, a VR theatre platform that integrates motion capture, and augmented reality with a distributed 3D gaming engine. The performance on the virtual stage will be projected in the MAGIC space. Selected audience members will have the opportunity to view the performance from within in the virtual space using an Oculus.

\section{A Letter Home}

\section{Ambarien Alqadar}

Borders between nations define who can travel where. A differential catalogue of the social, cultural and economic capital of the traveler determines who the traveler is and what she experiences. This VR project creates an immersive experience of American life for women in a small Indian beauty salon who've never set their foot on a plane.

\section{Music Viz}

\section{Zachary Glassner, Rudresh Pandit, Hongyu Zhao, Xuan Zhang}

This project aims to track the movements and the music of musicians to produce abstract art, which reflects the artist's vision. 


\section{LUNCHTIME DEMOS}

$12: 00-1: 00$

PerForM Lab in Imaging Science

(CAR - 2101)

Modeling human hand-eye coordination using VR

Kamran Binaee

Gulliver's Travels

Rakshit Kothari

Munsell Color Science Lab (MCSL)

(COL-1086)

Modeling Color Appearance in AR

Nargess Hassani

CIAS New Media Design Workshop

(BOO - 1320)

Experience Mindfulness

Anna Dining, Caleb Payne, Alec Burns

\section{AFTERNOON DEMOS}

$1: 45-5: 00$

\section{VIVE DEMOS (MAGIC VR Lab)}

\section{Virtual Marimba Performance}

Andrew Glaude, Eric Knapik, Martin Schuyler

$(1: 45-3: 00)$

The Virtual Marimba is a project using the HTC Vive that allows users to play a completely virtual percussion instrument. This project was created using the Unreal Engine and is sure to be a delight to try out!

\section{Cat Stack}

(3:00-4:00)

\section{Benjamin Connick}

Cat Stack is a silly and fun Virtual Reality game where you own a cat daycare that accepts too many cats. Keep your customers happy, even if you have to stack cats to the ceiling!

\section{Charlotte}

$(4: 00-5: 00)$

\section{Elizabeth Goins}

Charlotte is an exploration/walking simulator game that allows players to explore the history and culture of 19th century women through the short story, The Yellow Wallpaper and the life of its author, Charlotte Perkins Gilman. 


\section{OCULUS DEMOS (Coaching Room A in MAGIC Center)}

VR Photo Album

Arjun Dhuliya, Mychal Lipson, Nikhil Verma, Xiaoxuan Zhang

View a user's photo albums in a procedurally generated virtual environment where the user can walk into an art gallery with pictures hanging on walls.

\section{Visualizing Bioinformatics Datasets in VR (3:30 - 5:00)}

\section{Jimmy Zhang}

The GRIA-2 Protein in Virtual Reality. GRIA2 encodes a glutamate receptor important for fast neurotransmitter release. Harmful mutations in GRIA2 are associated with childhood intellectual disability etiology.

\section{BatmanVRSuperman}

$(3: 30-5: 00)$

\section{Aishwary Pramanik, Matthew Seita}

A well scripted VR game, suitable for all age groups, in which player can switch between Batman and Superman at any point of time, enjoy and tackle various difficulties in different levels.

\section{HOLOLENS \& VIVE DEMOS}

\section{VIVE Martial Arts Trainer}

\section{Nathan Stevens}

The goal of the application is to teach foundation martial arts movements, which can then be transferred to real world application and practice. The user will be taught the movements by receiving visual feedback from the application.

\section{Hololens 3D Locative Art}

\section{Sarathi Hansen, Khyati Vyas}

3D Locative Art is a shared augmented reality experience. The users can choose 3D models to place in the world and other users who are wearing a Hololens with the application running can view the model in the same location and same orientation.

\section{Shared Gaming}

Jeffrey Bauer, Mitayshh Dhaggai, Tanvi Raut, Niyati Shah

A framework built to support shared objects for users in different spaces using the Microsoft Hololens. A game of chess was built on this framework to demonstrate its capabilities. 
Realtime and offline measurement in a room used for saving room models.

\section{MS Hololens 3D Image Ear Training (3:30 - 5:00) \\ Justin Levine, Dr. Sungyoung Kim, Hiraku Okamura}

A new spatial ear-training paradigm is proposed using a see-through head-mount display that overlays target visual objects over auditory objects.

\section{POSTERS}

\section{Locative Art Mobile}

\section{Ajinkya Abhimanyu Dhaigude, Pratham Mehta}

Discover 3D art near you through our mobile app. Experience 3D augmented reality in familiar surroundings on the go!

AR applications for enhancing experiences and marketing services in the hospitality and tourism sector.

\section{Dr. Rick Lagiewski, Dr. Muhammet Kesgin}

An exploratory work into the best practices and coming trends in enhancing experiences and marketing services through AR in the hospitality and tourism industries. How tourism and hospitality providers are using AR to promote, attract and sell to consumers through mobile connections will be addressed.

\section{RocReadaR}

$(1: 45-3: 15)$

\section{Elena Fedorovskaya}

RocReadaR is a transmedia publishing platform designed and implemented to serve both readers and publishers. Readers use the application to seamlessly mingle between print and digital material of a publication and interact with 3D graphics using a smart phone.

Investigating integration between auditory and visual location information presented in an AR device.

$(1: 45-3: 15)$

Hiraku Okumura, Sushrut Khiwadkar, and Sungyoung Kim

Most of the AR devices use a technique called head related transfer function (HRTF). This technique will be demonstrated. 


\section{Anthony Zalar}

How to set up Google VR for video games. The presentation will prepare users for Android development with a focus on the Unity game engine.

\section{VR/AR Headset Design / DIY VR}

\section{Kim L Sherman, Ronald Vullo}

Junior Industrial Design students developed Virtual Reality Headsets for use with smart phones. At the MAGIC Center's virtual groundbreaking ceremony on November 15, the headsets were used by attendees to take a virtual tour of the proposed facility for the MAGIC center.

Manufacturing Work Instructions on Wearable and Mobile Devices with Augmented Reality

$(3: 30-5: 00)$

Dr. Michael Thurston, Scott Nichols

The project is to develop and demonstrate a "Shop Floor Digital Knowledge Transfer System" designed to provide augmented reality enabled electronic work instructions for manufacturing.

\section{ASL Interpreting through Smart Glass Technology (3:30 - 5:00)}

\section{Ashley Miller}

The addition of smart glasses technology as an accessibility tool could minimize this challenge for deaf and hard of hearing students in mainstream classrooms, addressing split visual attention by condensing three visual fields into one!

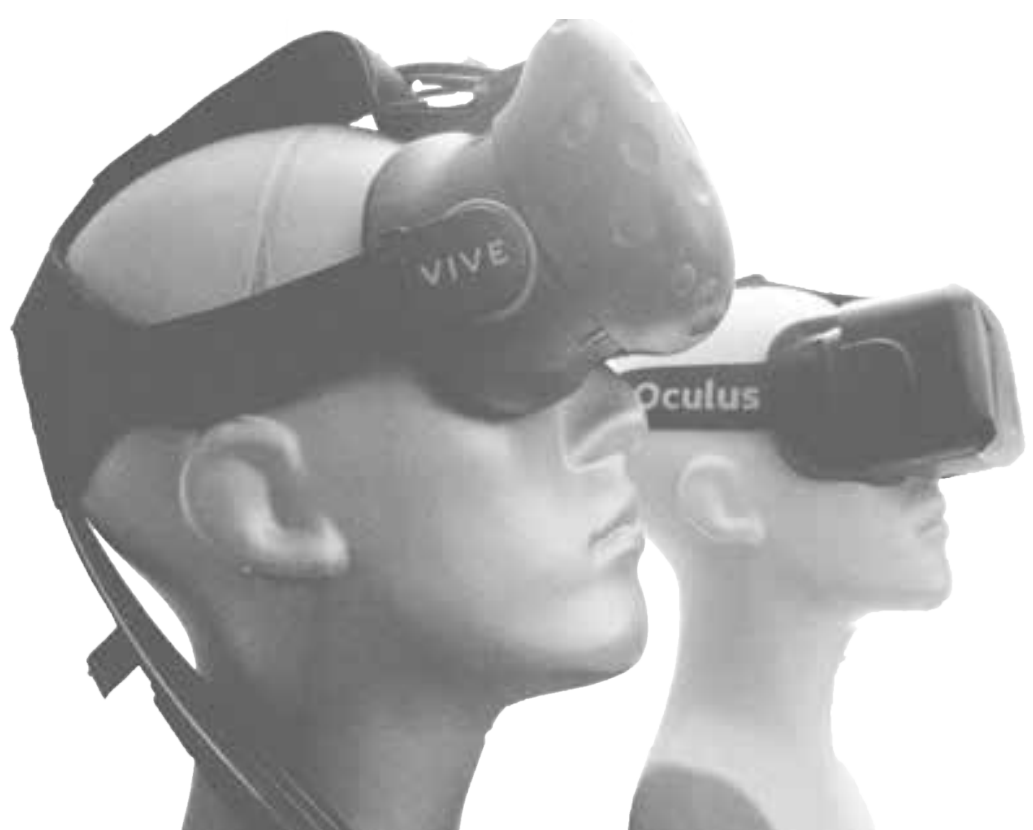




\section{FRAMELESS LABS VR/AR SYMPOSIUM}

\section{SYMPOSIUM CO-CHAIRS}

David Long

Joe Geigel

CIAS Motion Picture Science

GCCIS Computer Science

\section{SYMPOSIUM COMMITTEE}

Ambarien Alqadar

Reynold Bailey

Gabriel Diaz

Anna Dining

Ricardo Figueroa

Michael Murdoch

Peter Murphey

Caleb Payne

Mark Reisch
CIAS Film and Animation GCCIS Computer Science COS Imaging Science CIAS Motion Picture Science CIAS Motion Picture Science COS Color Science CIAS Film and Animation CIAS New Media Design CIAS Film and Animation

\section{EVENT HOSTS}
Andrew Phelps
Christopher Egert
Jennifer Hinton
Brenda Schlageter
RIT MAGIC Center
RIT MAGIC Center
RIT MAGIC Center
RIT MAGIC Center

\section{SPONSORS}

Dean's Office, GCCIS

Dean's Office, CIAS

RIT MAGIC Center

School of Film and Animation
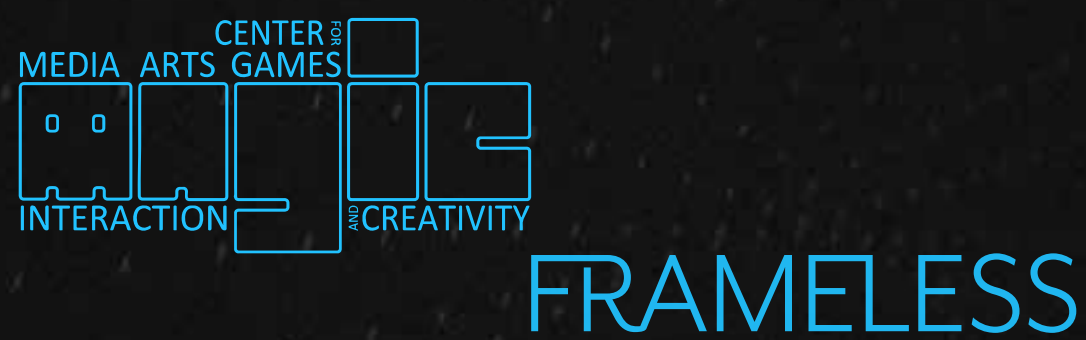